\title{
RESEARCH ON QUALITY FRAMEWORK OF REAL SCENE 3D MODEL BASED ON OBLIQUE PHOTOGRAMMETRY
}

\author{
Xu.Yongmin ${ }^{* 1}$, Zhang.Jixian, Zhao.Haitao, Chen.Chunxi, Mao.Wenjuan \\ National Quality Inspection and Testing Center for Surveying and Mapping Products , Number 28 Lianhuachixi Road, 100830 \\ Beijing,China-121176049@qq.com
}

Commission III, ICWG III/IVb

KEY WORDS: Real Scene 3D Model, Quality Framework, Quality Element, Position Accuracy, Texture Accuracy, Model Accuracy.

\begin{abstract}
:
With the development of real 3D model production technology and the expansion of application field, people pay more and more attention to the quality of real 3D model. However, how to measure the quality of today's real 3D model have been bothering its producers and users. In this paper, we analysed the quality model of real scene 3D model based on oblique photography from the perspective of the third party. Our analysis is guided by the application requirements of real scene 3D model, combined with the existing production technology level. Our analysis is guided by the application requirements of real scene 3D model, combined with the existing production technology level, we established the quality framework of real scene 3D model. This quality framework of real 3D model includes nine quality elements. Using this quality framework, we made a quality evaluation test in Yingjing County, Sichuan Provence. The test results show that the quality framework can fully reflect the quality of the real scene 3D model. The quality framework of real scene 3D model established in this paper solves the problem that it is difficult to evaluate the quality of real scene 3D model. The quality framework provides a basis for comprehensive and objective evaluation of real scene 3D model quality.
\end{abstract}

\section{INTRODUCTION}

With the development of oblique photogrammetry technology, real scene 3D model emerges as the times require. As a new type of basic surveying and mapping product, it has quickly won the favor of producers and users with the advantages of "all elements, true texture" holographic expression ability, high precision, abundant measurability, low cost, high efficiency constructability. However, how to measure the quality of today's real scene 3D model and what aspects need to determine the quality of real scene 3D model have been bothering its producers and users. In this paper, we analysed the quality model of real scene 3D model based on oblique photography from the perspective of the third party. Our analysis is guided by the application requirements of real scene 3D model, combined with the existing production technology level. Based on the analysis, we established the quality framework of real scene 3D model. Using this quality framework, we made a quality evaluation test. This evaluated 3D model is located in Yingjing County, Sichuan Province. The test results show that the quality framework can fully reflect the quality of the real scene $3 \mathrm{D}$ model. The quality framework of real scene 3D model established in this paper solves the problem that it is difficult to evaluate the quality of real scene 3D model. The quality framework provides a basis for comprehensive and objective evaluation of real scene $3 \mathrm{D}$ model quality.

\section{APPLICATION REQUIREMENT ANALYSIS OF REAL SCENE 3D MODE}

Real scene 3D model based on oblique photography has been widely used in urban management, natural resource management, smart city, emergency command, geological prevention and other fields.

2.1 Application requirements of natural resource
management

In the field of natural resources management, it is necessary to use real scene 3D model to assist planning and design. During the process of using, it is necessary to browse the appearance of 3D model in an all-round way. Position and height are important for them. They usually measure position and height. They carry out sunshine and inundation analysis based on the model. They add planned building, vegetation or other geographical elements to the 3D model with the help of special software or directly. They need to see the designed surface landscape on real scene 3D model.

\section{2 smart city}

A large amount of data is need in the construction and management of smart city. Oblique photogrammetry can obtain more data than traditional aerial photogrammetry. It can provide basic data for DSM, DOM, DLG and so on. These data is need in the construction of smart city.

In smart city management, model-based sunshine analysis, inundation analysis, collapse analysis and collision analysis are usually need.

\subsection{Emergency command}

Fast is the biggest characteristic of emergency command. Rapid acquisition of image data by oblique photogrammetry and rapid construction of real scene 3D model are the biggest requirements of emergency commanders. Through the real scene 3D model,

\footnotetext{
${ }^{1}$ Xu.YongminNational Quality Inspection and Testing Center for Surveying and Mapping Products , Number 28 Lianhuachixi Road, 100830 Beijing,China-121176049@qq.com
} 
the emergency commander can quickly know the situation of the disaster area at the first time. They need to analyze the disaster situation through model inundation, collapse and collision analysis. These analyses are helpful to make emergency plans.

\subsection{Geological prevention and control}

In order to do a good job in geology, geologists often need to measure the height, length, area, angle, slope and other aspects based on the model. The inundation and collapse analysis based on the model is an important analysis of the prevention of geological disasters.

Based on the summary and analysis of the application requirements of real scene $3 \mathrm{D}$ models in the above fields, we find that the application requirements of real scene $3 \mathrm{D}$ model in various fields mainly focus on the following aspects:

(1) Full range real-time displaying. The real scene 3D model should have the holographic expression ability of "all elements" and "true texture". It can truly reflect the appearance of ground objects and terrain and their mutual position relationship. It can help users to obtain immersive experience. It can provide convenience for users to understand the situation of the whole region.

(2) The real-time measurement. Users can directly measure the height, length, area, angle, slope and other aspects based on the real scene 3D model. It is helpful to provide real data for rapid decision-making.

(3) Three-dimension design. Users can add planned buildings, vegetation or other geographical elements to the real scene $3 \mathrm{D}$ model by means of special software or directly. The designed surface landscapea is reflected on the real scene 3D model directly. This function can provide assistance for design and planning decision-making.

(4) Three-dimension analysis. Users can analyze sunshine, submergence, inundation collapse and collision based on the model.

\section{QUALITY CHARACTERISTICS OF REAL SCENE 3D MODEL}

The real 3D model based on oblique photography is a kind of 3D tin network model with pyramid structure and texture. It is mostly stored in binary system and the data format is OSGB. It is very different from the traditional 3D model.

From the perspective of the application requirements of 3D models in various fields, there are many requirements for the position accuracy, model accuracy, scene effect, texture accuracy and time of 3D models. Combined with the characteristics of real scene 3D models, we can get the main quality characteristics of real scene 3D models, as shown in the table 1 .

\begin{tabular}{|l|c|}
\hline Quality characteristics & Quality description \\
\hline Plane position, & Accuracy \\
Elevation & \\
Shape & Integrity and correct \\
Texture & authentic and objective \\
3D Scene & Beautiful expression in \\
& all directions, Smooth \\
& browsing \\
Time & Newest and fresh \\
Auxiliary Tool & Comprehensive and \\
& easy \\
\hline
\end{tabular}

Table 1. Main quality characteristics.

The quality of real 3D model depends on the technical links of production. The production of real 3D model by using the data obtained from oblique photogrammetry mainly includes data acquisition, data pre-processing, joint adjustment of multi view images, dense matching of multi view images, DSM generation, texture mapping, auxiliary function development and other production links (as shown in Figure 1), This Production technology involving aviation, photography, Computer and other fields. The key production process determines the key quality of the real scene 3D model, so the key production process becomes the quality factor of the model.

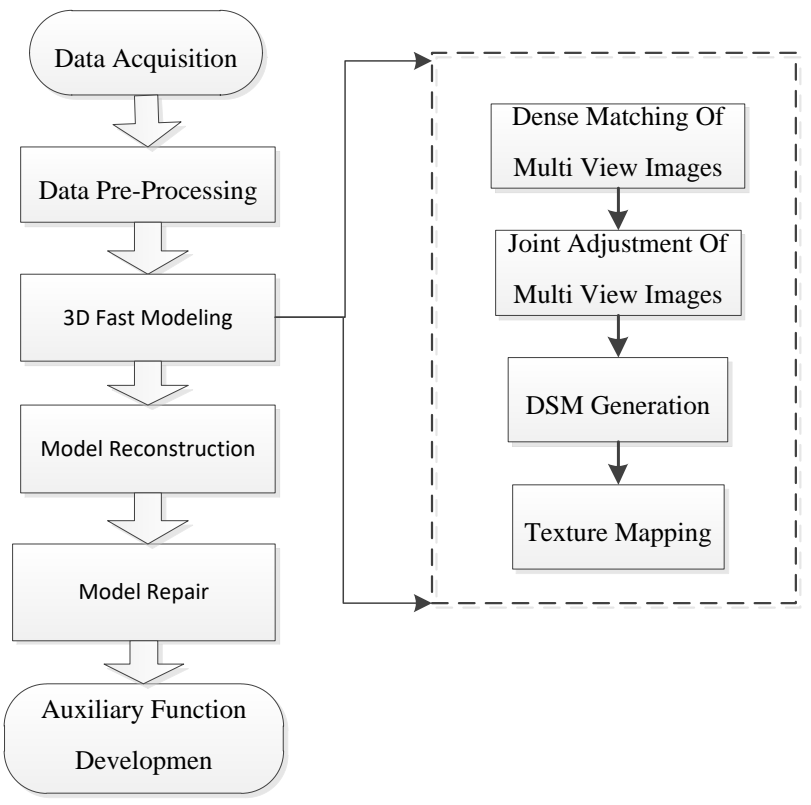

Figure 1. Key production process of real scene 3D model

In the production process of real scene 3D model, data acquisition is the basis link, and it is the basis of follow-up quality. Joint adjustment and matching of multi view images determine the accuracy of real scene 3D model. Texture mapping determines the authenticity and correctness of real scene 3D model, and it is the basis of 3D scene appearance. The development of 3D auxiliary tools is the guarantee of 3D analysis and design, and it is the key channels to the outward expression of real scene 3D model. Therefore, the main quality factors of real scene 3D model include data acquisition, joint adjustment of multi view image, dense matching of multi view image, texture mapping and auxiliary function development. 


\section{ANALYSIS AND CONSTRUCTION OF REAL SCENE 3D MODEL QUALITY MODEL}

The quality characteristics of real scene 3D models based on users' requirements are the basis of establishing the quality framework of real scene 3D models. The quality framework consists of multiple quality elements. From the perspective of quality measurement, we analysed the relationship between the quality elements and the quality characteristics. The relationship is shown in Table 2 .

\begin{tabular}{|l|c|}
\hline Quality elements & $\begin{array}{c}\text { Quality characteristics } \\
\text { Accuracy of plane } \\
\text { Tosition accuracy } \\
\text { position and elevation } \\
\text { Integrity and } \\
\text { correctness of shape } \\
\text { Authenticity and } \\
\text { objectivity of texture } \\
\text { Beautiful 3D Scene } \\
\text { Scene Effect }\end{array}$ \\
Time precision & Time is Newest \\
Integrity & gull expression of \\
& The three-dimensional \\
Usability & scene browsing is \\
& smooth, the three- \\
dimensional design & analysis function is \\
comprehensive and & easy to use \\
\hline
\end{tabular}

Table 2. The relationship between the quality elements and the quality characteristics.

From the perspective of quality measurement, in order to explain the accuracy of the plane and elevation of data, it is necessary to explain the plane and elevation of data from the same reference. Therefore, the spatial reference system of data is a quality element. The spatial reference system includes the reference of plane position and the reference of elevation. In order to introduce and explain the data situation and instructions of the real scene 3D model to the users, the technical documents and metadata matching with the real scene 3D model are essential, so the attachment quality is also the quality element to measure the real scene 3D model.

Through the above analysis, the quality framework to measure the quality of real scene 3D model data should include nine aspects: spatial reference system, position accuracy, model accuracy, texture accuracy, time accuracy, scene effect, integrity, usability and attachment quality.

In order to measure the quality better, the quality element includes multiple quality sub-elements. For example, the spatial reference system includes coordinate system, elevation datum and projection method. The specific quality framework of real scene 3D model is shown in Figure 2.

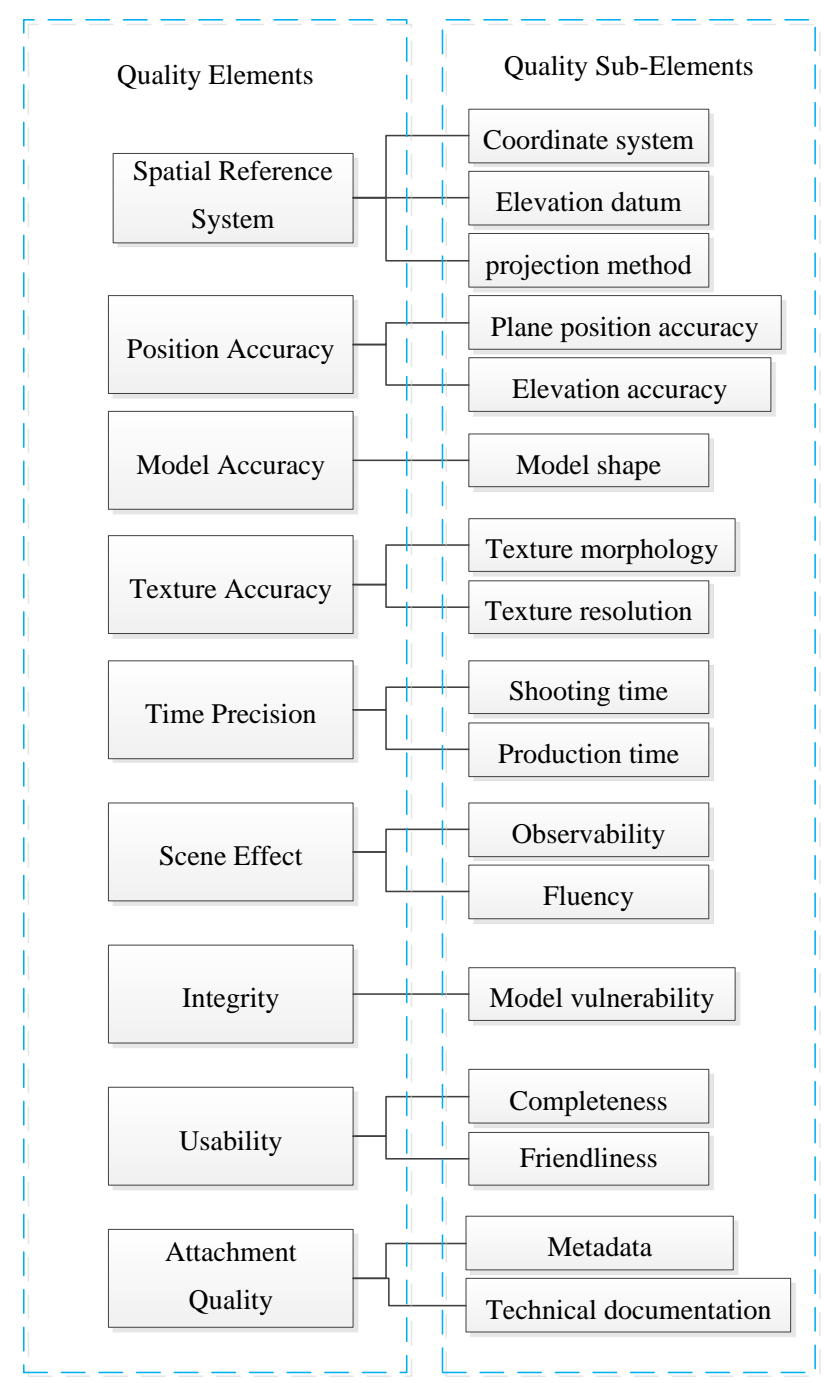

Figure 2. Real scene 3D model quality framework

In the quality measurement of real scene 3D model, each quality element represents different aspects, so each quality element have different evaluation method. In quality measurement, evaluation methods should be used according to the characteristics of each quality element or quality sub element. In the quality element evaluation of real scene 3D model, the mean square error, correctness, rationality, reliability and standardization are mainly used for evaluation. The specific evaluation method of each quality element is shown in Figure 3. 


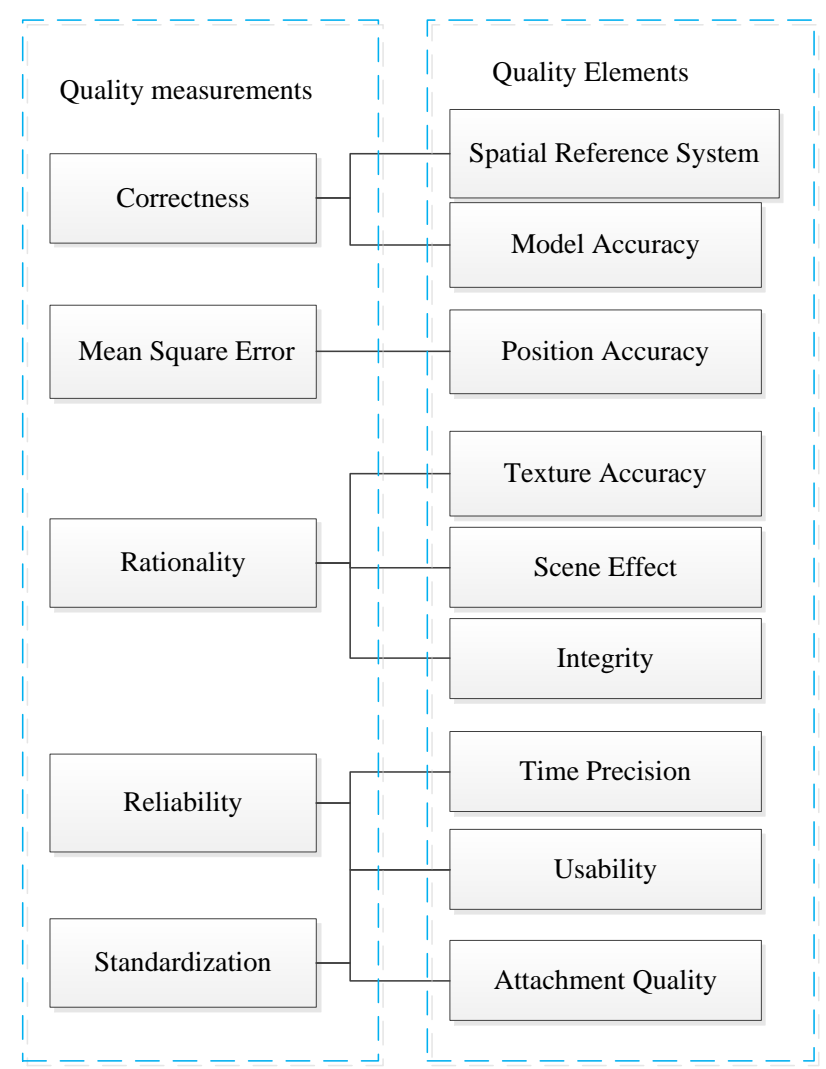

Figure 3. Real scene 3D model quality framework

The spatial reference system is the location reference of the realscale 3D model. The correctness of the spatial reference system determines the effectiveness of the real scene 3D model. Therefore, the spatial reference system is a veto. If the spatial reference system of the real scene $3 \mathrm{D}$ model is wrong, the real scene 3D model is judged as unqualified directly.

The position accuracy is evaluated by the mean square error of statistical detection points, and the position accuracy is evaluated by comparing the mean square error value of detection statistics with the mean square error value required by design or product standards. If the mean square error of the detection statistics is less than that of the product requirements, it is qualified. On the basis of qualified, the position accuracy can be judged according to the mean square error. If the mean square error value of detection statistics is greater than the mean square error value required by the product, it is unqualified.

Model accuracy is mainly used to measure the correctness of model shape. In real scene 3D models, a small number of complex models errors are allowed to have. Therefore, the model accuracy is evaluated by the error and leakage rate. If the error and leakage rate is lower than the allowable value required by the product, it is qualified. On the basis of qualified, the model accuracy can be judged according to the error and leakage rate. If the error and leakage rate exceeds the allowable value required by the product, it is unqualified.

Texture accuracy, Scene Effect and integrity are closely related to the shooting time and investment cost of the image, so these three quality elements can be measured by rationality. The accuracy of the evaluation rules is the same as that of the model, but the allowable value of the error and leakage rate will be different.
Time accuracy, usability of auxiliary software and quality of accessories are mainly measured by reliability and standardization. The time accuracy, the quality of auxiliary software and accessories are closely related to the user's requirements and uses. As long as they meet the user's requirements, they are qualified. If they do not meet the user's requirements, they are unqualified. General auxiliary software is allowed to have a small amount of deviation from the user's requirements, but the main functional requirements should be met. Similarly, the main indicators in the quality of accessories should be explained clearly.

Because the user's needs and the production technology requirements of the real scene $3 \mathrm{D}$ model will be clearly marked in the production design, the main basis for the quality evaluation of the real scene 3D model is the production design requirements of the real scene 3D model. However, the production design requirements should not be contrary to the national recommended product standards.

\section{APPLICATION TEST OF REAL SCENE 3D MODEL QUALITY FRAMEWORK}

Real scene 3D model quality framework test area is located in Yingjing County, Sichuan Province. Yingjing County is located in the south of 30 degrees north latitude. Its climate is subtropical climate. The terrain is high in the southwest and low in the northeast. It belongs to hilly landform. The buildings are mainly 1-2-storey brick buildings.

AC1100 six rotor UAV and ap4200r platform are used for data acquisition of real scene $3 \mathrm{D}$ model. The resolution of image acquisition is $0.015 \mathrm{~m}$, and the model results are stored in OSGB format. The coordinate system of the model results is WGS84, the elevation datum is geodetic elevation, and the projection is Gauss Kruger projection.

The quality inspection test used the quality framework designed in this paper. The position accuracy used comparison method. The detection points are obtained by total station and RTK, and the points with the same name on the model are collected manually.

51 plane detection points and 31 elevation detection points are collected. We used high-precision detection method to statistic position accuracy. The accuracy statistical formula is shown in Formula 1:

$$
\mathrm{M}=\sqrt{\frac{\sum_{i=1}^{n} \Delta_{i}^{2}}{n}}
$$

where $\quad \mathrm{M}=$ mean square error

$\mathrm{n}=$ Number of detection points

$\Delta_{i}=$ error, the field detection point is different from that of the same name point of the model. The formula is shown in Formula 2:

$$
\Delta_{i}=\sqrt{\Delta x^{2}+\Delta y^{2}},
$$

where $\Delta x=$ error, the field detection point is different from that of the same name point of the model, $\mathrm{x}$ direction

$\Delta y=$ error, the field detection point is different from that of the same name point of the model, $y$ direction

The mean square error of plane position is $0.037 \mathrm{~m}$, and the mean square error of elevation is $0.23 \mathrm{M}$. The distribution map of plane 
error is shown in Figure 4, and the distribution map of elevation error is shown in Figure 5.

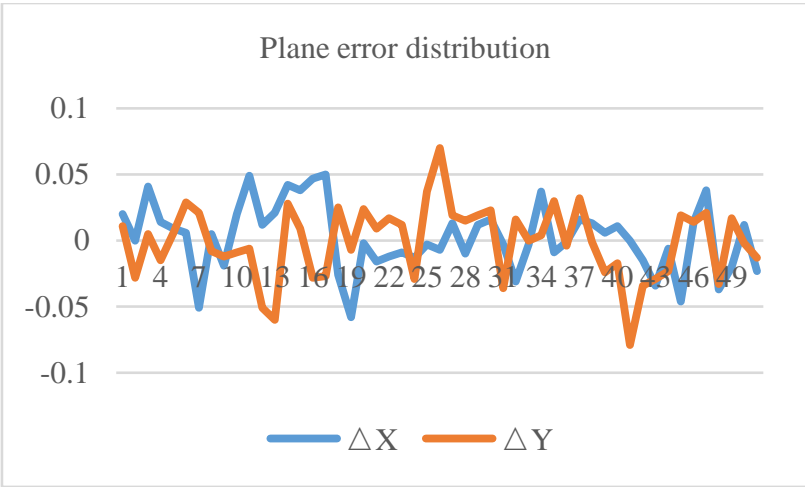

Figure 4. plane error distribution map

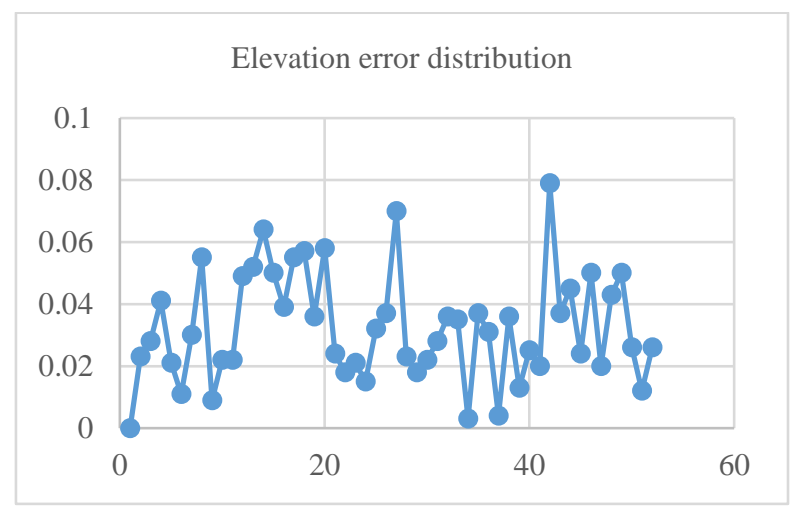

Figure 5. elevation, error distribution map

Other 8 quality elements, such as spatial reference system and model accuracy, are checked by human-computer interaction. Errors such as model structure deformation, texture deformation and model vulnerability are found. The error screenshots are shown in Figure 6, figure 7 and Figure 8, and the specific error number distribution is shown in Figure 9.

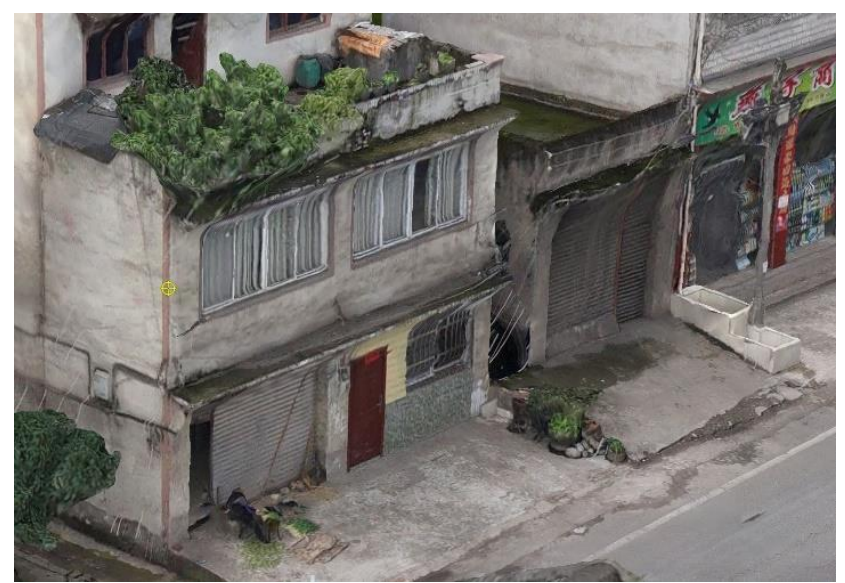

Figure 6. Model structure deformation

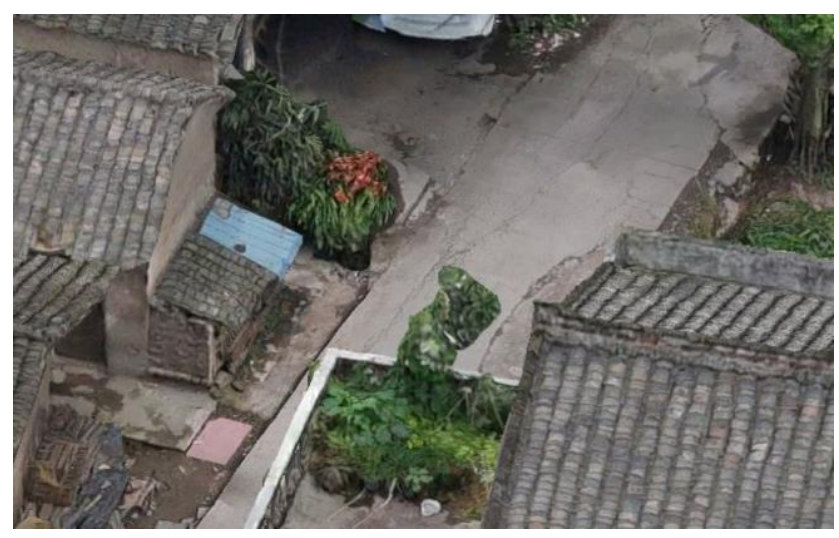

Figure 7. texture deformation

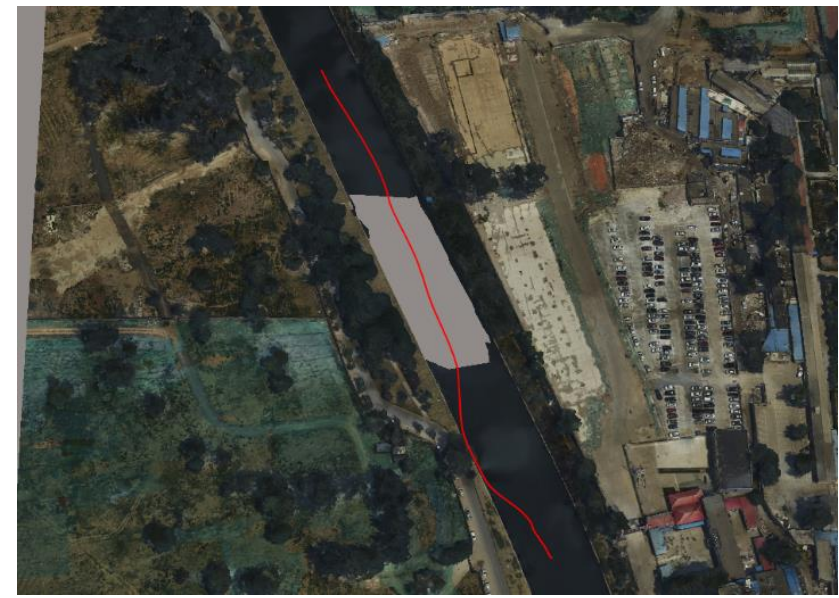

Figure 8. Model vulnerability

\section{Error number distribution}

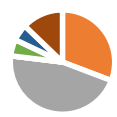

$$
\begin{array}{ll}
\text { - } \text { spatial reference system } & \text { - model accuracy } \\
\text { " Texture accuracy } & \text { - Integrity } \\
\text { - Time precision } & \text { - Scene Effect } \\
\text { - usability } & \text { - attachment quality }
\end{array}
$$

Figure 9. elevation error distribution map

The test results of quality inspection can reflect the quality of the real scene 3D model, and the inspection results are consistent with the quality expectations of producers and users.

\section{CONCLUSION}

In this paper, guided by the application of real scene 3D model, combined with the characteristics of real scene 3D model and the existing, more mature production technology capacity, we established a framework to measure the quality of real scene $3 \mathrm{D}$ model. The establishment of the quality framework solves the problem that there is no basis for the quality evaluation of real 
scene 3D model, and lays the foundation for the comprehensive and objective measurement of the quality of real scene 3D model.

\section{REFERENCES}

Ku. J. S. Lee, K. M. Lee, 2001a:Multi-image Matching for a General Motion Stereo Camera Model.PaREC, Vol.34, pp.1701$1712,2001$.

Lei Lei, Xu Jing Yan, 2018a: Texture refinement technology of 3D model based on oblique photogrammetry. Standardization of Surveying and mapping, 2018, vol34 (1), pp. 56-58.

Li Yingjie, 2016b, Research on aerial oblique multi view image matching method. Beijing: China Academy of Surveying and mapping.

Li Zhenzhou, Zhang Xuezhi, 2012a. Research on the Quick Construction of 3D Model of City Based on Oblique Photogrammetric Technique. Geomatics \& Spatial Information Technology, 35(04), pp.117-119.

National Administration of Surveying, Mapping and Geoinformation. CH/T9015-2012, Specifications for the Digital Products of Three-Dimensional Model on Geographic Information. Surveying and Mapping Press, 2012.

National Administration of Surveying, Mapping and Geoinformation. CH/T 9024-2014, Specifications for Inspection and Acceptance of Quality of Three-Dimensional Model Data Products on Geographic Information. Surveying and Mapping Press, 2014

Yang Xinli, 2017a. Study on 3D Digital City Modeling on the Basis of Oblique Photography Measurement Technology. Intelligent City, (02), pp.5.

Zhou Xiaomin, Meng Xiaolin, Zhang Xueping, 2016a. Construction method of urban true 3D model based on oblique photogrammetry. Surveying and Mapping Science, , vol41 (9): $159-163$

Zhou Jie, 2016a. Research on the Method of Constructing Real scene 3D Model Based on Oblique Photogrammetry Technology. Value Engineering, 35(25), pp.232-236.

YANG Guo-dong, WANG Min-shui, 2016a. The Tilt Photographic Measuration Technique and Expection. Geomatics \& Spatial Information Technology,39(1), pp.13-15. 